\title{
Design and realization of the ZigBee wireless sensor network experimental platform
}

\author{
Di Qu ${ }^{1, a}$, Yuanyu Sun² \\ ${ }^{1}$ China Telecommunication Technology Labs - Terminals, China Academy of Information and \\ Communications Technology, Beijing 100191, China \\ ${ }^{2}$ Department of Business Development, China Academy of Information and Communications \\ Technology, Beijing 100191, China \\ aselina.q@163.com
}

\begin{abstract}
Keywords: ZigBee; wireless sensor network; network experimental platform; design and realization Abstract. In the current society, with the rapid development of science and technology, many new techniques have found increasingly extensive applications in various fields. The ZigBee wireless sensor network experimental platform is a representative technique, which has shown favorable functions and results during the practical application process. Based on that, this paper studies design and realization of the ZigBee wireless sensor network experimental platform. First, basic components and functions of the network experimental platform are analyzed. Then, the hardware structure and software design of the equipment are analyzed. This research is an attempt to boost further development and promotion of techniques in the field.
\end{abstract}

\section{Introduction}

In the current society, the wireless sensor network has found wide applications. Lots of intelligent sensor nodes have formed a self-organizing network through the wireless multi-hop method and been widely applied to the field of smart home, environment monitoring, trans-interaction, medical care, agriculture, industry and military. Based on the protocol specifications, the emerging wireless network technique, ZigBee, has been developed. Compared with WIFI, Bluetooth and other techniques, ZigBee is more suitable for the low-speed WSN, and can well meet requirements of power consumption, dynamic networking, safety and cost. Therefore, the ZigBee wireless sensor network experimental platform has shown favorable results in practical applications.

\section{Components and functions of the experimental platform}

In the wireless sensor network experimental platform based on ZigBee, the major system components and functions are shown in Table 1, which include the upper computer for software operation of ZigBee Center and the ZigBee electricity-saving equipment, such as the sensor terminal, the router and the coordinator. The network is built by the coordinator. Terminals like the sensor and the router are switched into it. According to relevant requirements, the on-site parameters are collected by the sensor and are concentrated to the coordinator through the network made up of several routers. Then, the upper computer receives the data forwarded by the coordinator. The ZigBee Center software classification is adopted to display, process and store these data [1]. Besides, in the upper computer, the coordinator can be used to realize monitoring and control of the node health conditions and the network dynamic topological structure. The platform can be used to build the ZigBee WSN. (See Fig. 1) In order to endow the experimental platform with favorable equipment universality, the hardware structural design should adopt the modularized structure, in which the operation of routers and coordinators is supported by the main control panel hardware. The main control panel is connected to the sensor panel with different functions so as to form the sensor terminal.

Table 1 Components and functions of the experimental platform 


\begin{tabular}{|c|c|c|}
\hline 1 & Upper computer & Operate the interface \\
\hline 2 & ZigBee coordinator node & $\begin{array}{c}\text { Realize communication between the upper computer and the terminal node } \\
\text { through the serial interface }\end{array}$ \\
\hline 3 & ZigBee terminal node & $\begin{array}{l}\text { Realize communication between the coordinator node and the terminal } \\
\text { node through the } 2.4 \mathrm{MHz} \text { frequency band signals }\end{array}$ \\
\hline 4 & Data collection terminal & Confirm the position of obstacles \\
\hline
\end{tabular}

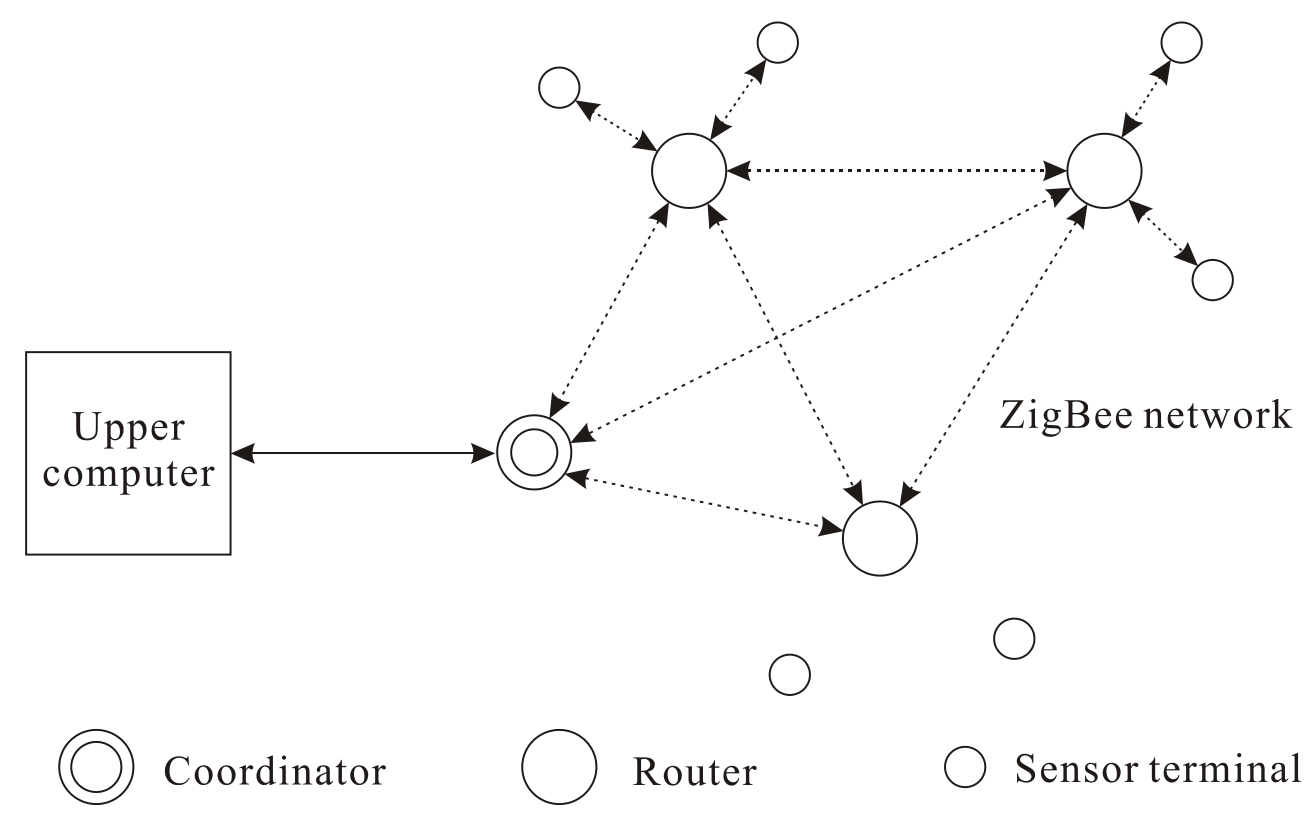

Fig. 1 ZigBee WSN schematic diagram

\section{Hardware structure of the experimental platform}

2.1 Main control panel. In the main control panel, there is the wireless micro-controller, the serial Flash memorizer, the serial interface chip, the extension socket and the basic man-machine interface. The specific hardware structure is shown in Fig. 2. Among them, the wireless micro-controller is a 32-bit third-generation wireless micro-controller, which includes 32-bit CPU, $2.4 \mathrm{MHz}$ radio frequency transceriver, $128 \mathrm{~Kb}$ red-only memory and random access memory, five-channel SPI, one digital audio interface, two asynchronous serials, two analog comparators, 21-channel interface, 2-channel 12-bit DAC, three timers and counters, four-channel 12-bit ADC, three system timers, watchdog and two-wire serial compatible with I2C/Smbus [2].

In the external serial Flash memorizer, the ZigBee application program codes designed for users are stored. After the system is restored, the read-only memory is used to add load to the random access memory. Among them, the USB interface and the asynchronous interface " 0 " of the upper computer realize adaptation connection and functions, including power supply of the main control panel, the uploading of data, the downloading of codes, etc. Besides, the main control panel can be supplied with power in the form of the voltage-stabilized source and battery. In the experimental platform, there is one passive buzzer, two buttons, two LEDs and so on, which jointly constitute the basic human-machine interface, so certain model can be set and the status of the main control panel can be displayed. Besides, the external extension sensor is realized through two 20-pin sockets. Among the extension signals, apart from the standard SPI and 21-channel parallel interface, there are reset signals, two-channel DAC and four-channel ADC [3]. 


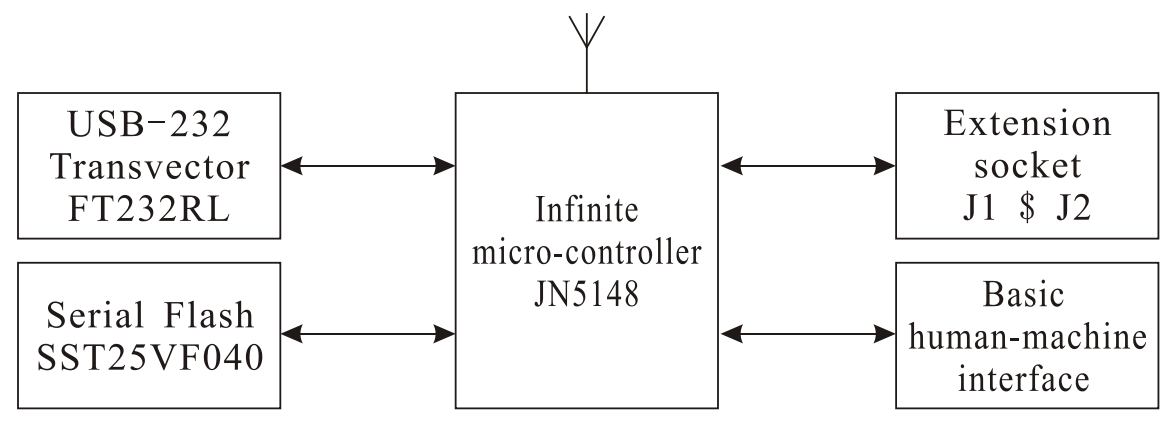

Fig. 2 Hardware structure of the main control panel

2.2 Sensor panel. In the experimental platform, there are different kinds of sensor panels. For example, the S01 sensor panel is a major application form. Its specific circuit structure chart is shown in Fig. 3. AT24C01A is the $1 \mathrm{~Kb}$ serial E2PROM memorizer of the I2C interface to store the equipment ID constituted of the 32-bit sequence code and the 16-bit type code. In JN5148, when the bus reuse port of I2C is DIO14 and DIO15, then it can be directly connected with SDA and SCL. In the low-cost monobus, DHT11 is the temperature and moisture sensor module, which can measure temperature ranging from $0{ }^{\circ} \mathrm{C}$ to $50^{\circ} \mathrm{C}$. Besides, it can reach the measurement recognition rate of $1^{\circ} \mathrm{C}$ and the measurement accuracy around $2^{\circ} \mathrm{C}$. Between $20 \%$ of RH and $90 \%$ of RH, the moisture can be measured and the measurement can reach $1 \%$ of the $\mathrm{RH}$ resolution ratio and $5 \%$ of the $\mathrm{RH}$ resolution ratio. The pyroelectricity infrared induction module is PIR. During the output process, the high-low level is adopted. Within the visual perspective of $100^{\circ}$, the effective operating distance can reach $5 \mathrm{~m}$ to $7 \mathrm{~m}[4]$.

In the camera module, the processor and the imaging sensor is equipped with built-in. The external commands can control it, conduct real-time image collection and finish the serial output, photo compression and other tasks as well. In C328, the data transmission format should be compatible with RS232, thus can reach the maximum Baud rate of $115.2 \mathrm{~Kb}$ per second. Besides, it has the 3.3v signal level, so it can be connected with the wireless micro-controller serial. There are six infrared LEDs. If the lighting is inadequate, the fill-in light will be introduced to control its lightness through the micro-controller. The 5516-type photoresistor with 500 kilo-ohm dark resistance and 10 kilo-ohm light resistance in the lighting sensor is adopted. After the pull-down resistor conducts partial pressure of it, the analog voltage will be output according to changes of lightness, and the tail-end controller controls its DC current transfer. In the magnetic door contact, the reed switch is applied. Based on the function of the pull-up resistor, the high-low level is output and the switch status is indicated.

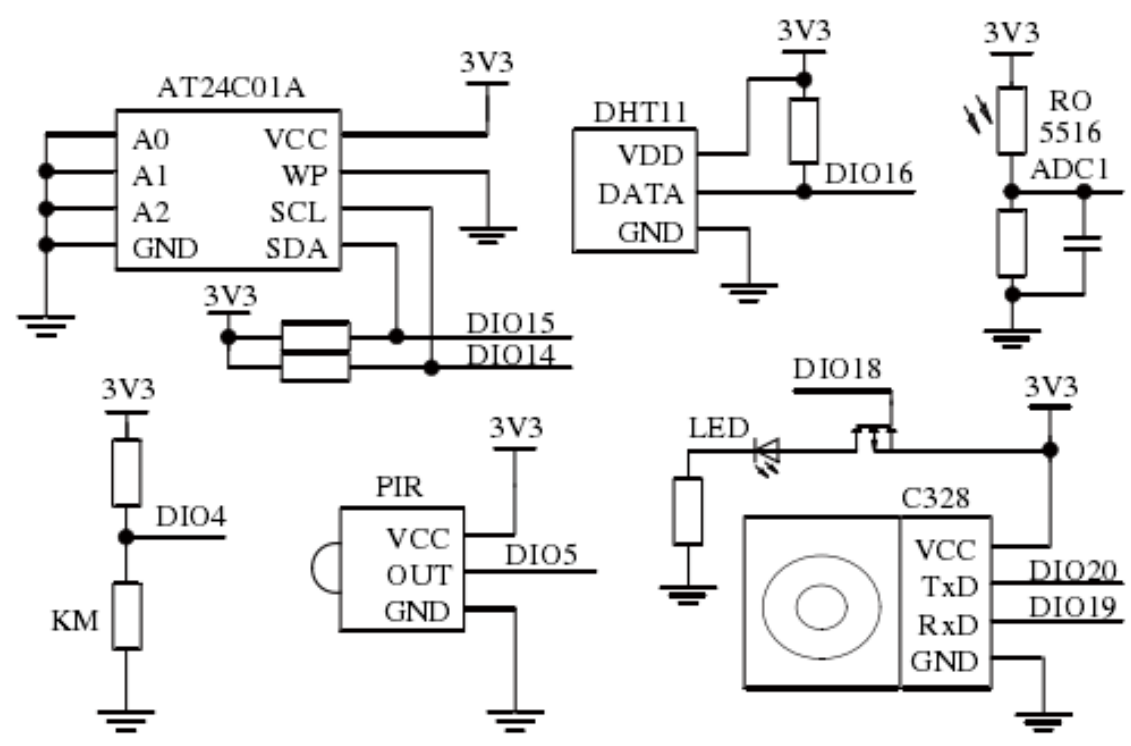

Fig. 3 Circuit structural chart of the sensor panel 


\section{Software design of the experimental platform}

Under the support of the hardware platform, there are three experiments of different layers, including WSN design experiment, data collection comprehensive experiment and wireless micro-controller interface experiment. In the second and third experiments, through the corresponding API function, the control and driving sensor, the executing equipment and the onboard human-machine interface equipment can be designed. In the first experiment, the corresponding protocol stack API function and the operation system should be used to design the coordinator, the router and the sensor terminal so as to finish the data convergence treatment, the wireless router transmission and the distributed collection [5]. Among them, the design of the router program and the coordinator program is relatively easily. In the user program, major tasks include operation status indication, network initialization, operation system and equipment accomplishment, and serial communication between the upper computer and the coordinator.

However, in terms of the sensor terminal, the program is relatively complex. After system initialization, the sensor terminal joins the network. According to the set interval of the upper computer, the terminal sensor conducts regular sampling of the sensor output. Besides, the APDU data frames are assembled and loosened according to the corresponding function. The major status of the sensor terminal is SLEEP, which can reduce the average current. Under the condition of the DIO terminal or the Wake Timer overflowing, the sensor will be aroused. It is confirmed whether the upper computer commands sent from the coordinator are father nodes. If the sampling conditions are met, data sending, assembly and collection can be conducted. Then, the SLEEP status can be resumed. Among the development tools for the ZigBee PRO equipment, there is the configurator, the debugging server, the Flash programming unit, C-language compiler and the corresponding integrated environment. $\mathrm{C}++$ Builder can be adopted to develop the ZigBee Center of the upper computer, and apply the serial event driving program structure.

\section{Conclusions}

ZigBee wireless sensor is a very important technique. The wireless sensor network experimental platform based on the ZigBee is one of its applications. During the practical application process, the network experimental platform has shown favorable effects and functions. Besides, it has found extensive applications in various fields. During the design and realization process of the ZigBee wireless sensor network experimental platform, corresponding techniques and equipment can be used so as to give full play to its functions and enable it to provide better services in relevant fields.

\section{References:}

[1]YU Tong, WANG Wenxiu \& XU Xiaozhan. Study of mobile videos delivery based on HTTP protocol[J]. Computer Technology and Development, 2012, 07: 167-170.

[2]TAO Weige, CHEN Xingrui, JIA Zhongning \& QIANG Zhiwen. Construction of a WSN teaching experimental platform based on ZigBee[J]. Research and Exploration in Laboratory, 2012, 12: 231-234.

[3]SHI Jianguo, MA Yunhui, ZHANG Huafu \& SHI Xiangqun. Development of the wireless sensor network teaching experimental platform base on ZigBee[J]. Internet of Things Technologies, 2013, 06: 28-30.

[4]XU Junxia \& XU Ke. Design and realization of the wireless sensor network nodes based on IEEE802.15.4 ZigBee[J]. Journal of Chengde Petroleum College,2011,02:57-60.

[5]KONG Danfeng \& ZHU Yongping. Design and realization of the wireless sensor network platform based on the ZigBee[J]. Journal of Changsha Telecommunications and Technology Vocational College, 2015, 02: 16-19. 